\title{
Impact of central venous pressure on the mortality of patients with sepsis-related acute kidney injury: a propensity score-matched analysis based on the MIMIC IV database
}

\author{
Yan Huo ${ }^{1 \#}$, Xinrui Wang ${ }^{1 \#}$, Bo $\mathrm{Li}^{1}$, Jordi Rello ${ }^{2}$, Won Young Kim ${ }^{3}$, Xiaoting Wang ${ }^{4}$, Zhenjie $\mathrm{Hu}^{1}$ \\ ${ }^{1}$ Department of Intensive Care Unit, Hebei Medical University Fourth Affiliated Hospital and Hebei Provincial Tumor Hospital, Shijiazhuang, \\ China; ${ }^{2}$ Clinical Research/Epidemiology in Pneumonia and Sepsis, Vall d'Hebron Institute of Research, Barcelona, Spain \& Clinical Research, CHU \\ Nîmes, Nimes, France; ${ }^{3}$ Department of Emergency Medicine, University of Ulsan College of Medicine, Asan Medical Center, Seoul, Republic \\ of Korea; ${ }^{4}$ Department of Critical Care Medicine, Peking Union Medical College Hospital, Peking Union Medical College, Chinese Academy of \\ Medical Sciences, Beijing, China \\ Contributions: (I) Conception and design: Y Huo, Z Hu; (II) Administrative support: None; (III) Provision of study materials or patients: All authors; \\ (IV) Collection and assembly of data: Y Huo, Xinrui Wang, B Li; (V) Data analysis and interpretation: Y Huo, B Li; (VI) Manuscript writing: All \\ authors; (VII) Final approval of manuscript: All authors. \\ "These authors contributed equally to this work. \\ Correspondence to: Prof. Zhenjie Hu, MD. Department of Intensive Care Unit, Hebei Medical University Fourth Affiliated Hospital and Hebei \\ Provincial Tumor Hospital, 12 Jiankang Road, Shijiazhuang, China. Email: syicu@vip.sina.com.
}

Background: Sepsis has long been a life-threatening organ dysfunction. Sepsis associated acute kidney injury (SA-AKI) is an important complication of sepsis, as an important hemodynamic index, the impact of central venous pressure (CVP) on sepsis patients needs to be explored. Thus this study aimed to investigate the relationship between CVP and the mortality of SA-AKI.

Methods: Clinical data of adult patients with sepsis-related acute kidney injury, defined as met both the Sepsis 3.0 criteria and the Kidney Disease Improving Global Outcomes Clinical Practice Guideline (KDIGO) criteria, were obtained from the Medical Information Mart for Intensive Care-IV (MIMIC-IV) database. The included cohort was divided into a high CVP and a low CVP group were determined based on the cuf-off value from receiver operating characteristic curve, with propensity score-matched analysis of the 28-day mortality for both groups and sensitivity analysis using inverse the probability-weighting model, multifactorial regression, and doubly robust estimation, patients acquired chronic coronary syndrome (CCS) and diabetes were also taken into consideration.

Results: Of 1,377 patients with sepsis-related acute kidney injury, low CVP group $(<13 \mathrm{mmHg})$ was $67.4 \%(n=928)$ and high CVP group $(\geq 13 \mathrm{mmHg})$ was $32.6 \%(\mathrm{n}=449)$. The two groups were matched 1:1 by propensity score to obtain a matched cohort $(\mathrm{n}=288)$. The mortality rates in the low versus high CVP group (19.4\% vs. 34.7\%) were statistically difference (odds ratio OR: $0.454 ; 95 \%$ confidence interval 0.263 , 0.771). Moreover, the bistable analysis of logistic regression of the matched cohort (OR: $0.434 ; 95 \%$ CI: 0.244, 0.757), propensity score inverse probability weighting (IPW) (OR: 0.547; 95\% CI: 0.454, 0.658), and multifactorial logistic regression (OR: 0.352; 95\% CI: 0.127, 0.932) all yielded the same results.

Conclusions: In patients with sepsis-related acute kidney injury, a lower CVP level $(<13 \mathrm{mmHg})$ is an independent variable associated with decreased mortality. The threshold of CVP needs to be controlled in clinical work to improve the prognosis of patients with SA-AKI.

Keywords: Sepsis; mortality; critical care; database; central venous pressure (CVP)

Submitted Nov 23, 2021. Accepted for publication Feb 21, 2022.

doi: $10.21037 /$ atm-22-588

View this article at: https://dx.doi.org/10.21037/atm-22-588 


\section{Introduction}

Sepsis, which is caused by infection, has long been a lifethreatening organ dysfunction (1). More than 30 million patients are affected each year, with approximately 5.3 million deaths (2). The overall sepsis-related mortality has been stable for this time-period in both males and females (3). Owing to the publishing of guidelines for sepsis and the improvement of therapy, the grim situation has been eased (4), but there are still some questions about sepsis need to be addressed (5). Sepsis-related acute kidney injury (SA-AKI) is a life-threatening complication in critically ill patients (6), which is often combined by multiorgan dysfunction, and its outcome is mostly associated with a poor prognosis in critically ill patients (7). If not treated properly, it often leads to unrecoverable kidney damage, which can progress to chronic renal failure (8). Common risk factors for AKI include advanced age, chronic kidney disease (CKD), and cardiovascular disease. Since SA-AKI patients are often included in the AKI patient population, these factors may also be risk factors for poor prognosis in SA-AKI (9). In the absence of a unique treatment, the key to SA-AKI therapy lies in the promptness of diagnosis (10), as well as the reduction of renal burden by improving systemic hemodynamic status to avoid further exacerbation of renal injury (11).

SA-AKI is often accompanied by transient renal function failure (12), with a six- to eight-fold increase in in-hospital mortality of SA-AKI patients, in addition to the fact that more than $25 \%$ of patients with SA-AKI require renal replacement therapy (RRT) (13). Published in 2012, the Kidney Disease Improving Global Outcomes (KDIGO) criteria are the most widely accepted international diagnostic criteria for acute kidney injury (AKI) (14). Previous study had shown that the 1 -year mortality rate of patients recovering from SA-AKI was similar to that of patients without AKI (13), demonstrating that kidney injury caused by SA-AKI can be reversed with timely intervention (15). Central venous pressure (CVP), a volume index, is an important hemodynamic factor in the development of AKI, and previous reports have suggested that high levels of CVP often have a negative effect on the prognosis of AKI (16). However, research on the effect of CVP on the prognosis of SA-AKI is lacking, and given the different grading of SA-AKI in the KDIGO guidelines, the effect of CVP on different levels of AKI remains to be clarified. The purpose of this study was to explore the effect of different levels of CVP on the prognosis of SA-AKI with different KDIGO grading.
We present the following article in accordance with the STROBE reporting checklist (available at https://atm. amegroups.com/article/view/10.21037/atm-22-588/rc).

\section{Methods}

\section{The Medical Information Mart for Intensive Care IV (MIMIC-IV) database}

The MIMIC-IV is a database which can get access to for free, and contains information from 2008 to 2019 (17) (https://doi.org/10.13026/s6n6-xd98). The database records hourly physiological readings from bedside monitors verified by intensive care unit (ICU) nurses, and also contains data on demographics, laboratory indicators, nursing records, intravenous medications, fluid balance, and other clinical variables. The MIMIC-IV database is accessible to individuals who have completed the Collaborative Institutional Training Initiative examination. The study was conducted in accordance with the Declaration of Helsinki (as revised in 2013).

\section{The Kidney Disease Improving Global Outcomes (KDIGO) guideline}

AKI was defined as the KDIGO criteria of a $0.3 \mathrm{mg} / \mathrm{dL}$ change in serum creatinine within 48 hours or a $50 \%$ increase from baseline creatinine. For patients with serum creatinine within 7-365 days prior to admission, the most recent serum creatinine value was considered the baseline creatinine. For patients with missing baseline creatinine, the baseline eGFR was assumed to be $75 \mathrm{~mL} / \mathrm{min}$ per $1.73 \mathrm{~m}^{2}$ as estimated by the Modified Renal Disease Diet (MDRD) study equation according to the KDIGO AKI guidelines.

\section{Introduction to the study}

This is a longitudinal retrospective study. The MIMICIV database contains a total of 257,366 individuals, 35,010 of which were diagnosed with sepsis (as defined by the Sepsis-3 criteria) (1). In this study, we collected the data of each patient who was first admitted into ICU. The maximal blood creatinine level each day and the weight-normalized urine output every $6 \mathrm{~h}$ were collected according to the KDIGO classification. We designed this trial to explore the relationship between CVP levels and clinical outcomes in patients with different grades of SA-AKI, and to explore whether controlling CVP at certain levels contributes to 
improvements in mortality and benefit the management of septic patients in ICU.

\section{Inclusion and exclusion criteria}

Patients diagnosed with AKI according to the KDIGO criteria within 24 hours before or after sepsis diagnostic were enrolled in this study. The exclusion criteria were as follows: (I) patients aged $\leq 18$ years; (II) patients who did not meet the KDIGO criteria; (III) patients who were not admitted to the ICU for the first time; (IV) patients admitted to a care unit other than the medical ICU and surgical ICU; (V) received contrast therapy within 48 hours before or after diagnosis of acute kidney injury; (VI) patients for whom CVP data were not collected.

\section{Outcome}

The outcome indicator for the study was mortality at 28 days after meeting the enrolment criteria.

\section{Statistical analysis}

Patients were divided into high and low CVP groups by performing receiver operating characteristic (ROC) analysis of the post-enrolment CVP levels of the study population (18), which resulted in cut-off values. Propensity scores were calculated for the high CVP group versus the low CVP group using a gradient boosted model (GBM), which in turn minimized the differences between the covariates of the two groups. A matching cohort was obtained by 1:1 matching based on the propensity score exchangers of the patients. The relationship between CVP and patient prognosis was explored in the matched cohort using a bivariate robust analysis of a multifactorial regression combined propensity score model. An inverse probability weighting (IPW) model was used to generate a weighted cohort using estimated propensity scores as weights, and logistic regression was performed on the weighted cohort.

Since the propensity score model had balanced the two comparison groups, we compared the imbalance of the covariates between the original and adjusted (weighted) cohorts to assess the effectiveness. Standardized mean differences (SSMDs) between the high and low CVP groups were calculated. The non-parametric Wilcoxon signed rank sum test was used to test for statistical differences between continuous covariates. The chi-square test was used to test the relationship between categorical covariates. $\mathrm{P}<0.05$ was considered to indicate a statistically significant difference on both side.

\section{Covariates}

We took gender, age, height, weight, and the severity of patients (measured by the first 24-h Sequential Organ Failure Assessment (SOFA score), Glasgow Coma Scale Score (GCS score), and Charlson Score) into consideration cause these factors may leads to poor prognosis in SA-AKI.

\section{Comorbidities}

We extracted data on comorbidities such as CKD, congestive heart failure, atrial fibrillation, chronic liver disease, chronic obstructive pulmonary disease (COPD), CCS, hypertension, diabetes, stroke, and malignancy. All of these factors were identified on the basis of the recorded ICD-9 codes.

\section{Vital signs}

The heart rate, temperature, CVP, and mean arterial blood pressure (MAP) were collected as vital signs. The values for vital signs were averaged over a 24-hour period before and after meeting the definition of sepsis.

\section{Interventions}

Treatment like continuous renal replacement therapy (CRRT), mechanical ventilation, vasopressor, sedation, and analgesia were also collected in our study.

\section{Laboratory results}

Parameters including white blood cell (WBC), hemoglobin, platelet, albumin (ALB), total bilirubin (Tbil), creatinine (Cre), blood urea nitrogen (BUN), lactate, oxygenation index $(\mathrm{P} / \mathrm{F}), \mathrm{PCO}_{2}$, Troponin, brain natriuretic peptide (BNP) and creatinine kinase were considered in our study. We noted that more than half of the patients had missing troponin assays, and if we had used the troponin values directly as covariates, then there would be a large number of missing values. Therefore, we used whether troponin was collected as a covariate in the model, and similarly, there were more missing values for BNP and creatine kinase assays, and we took the same means to determine whether 


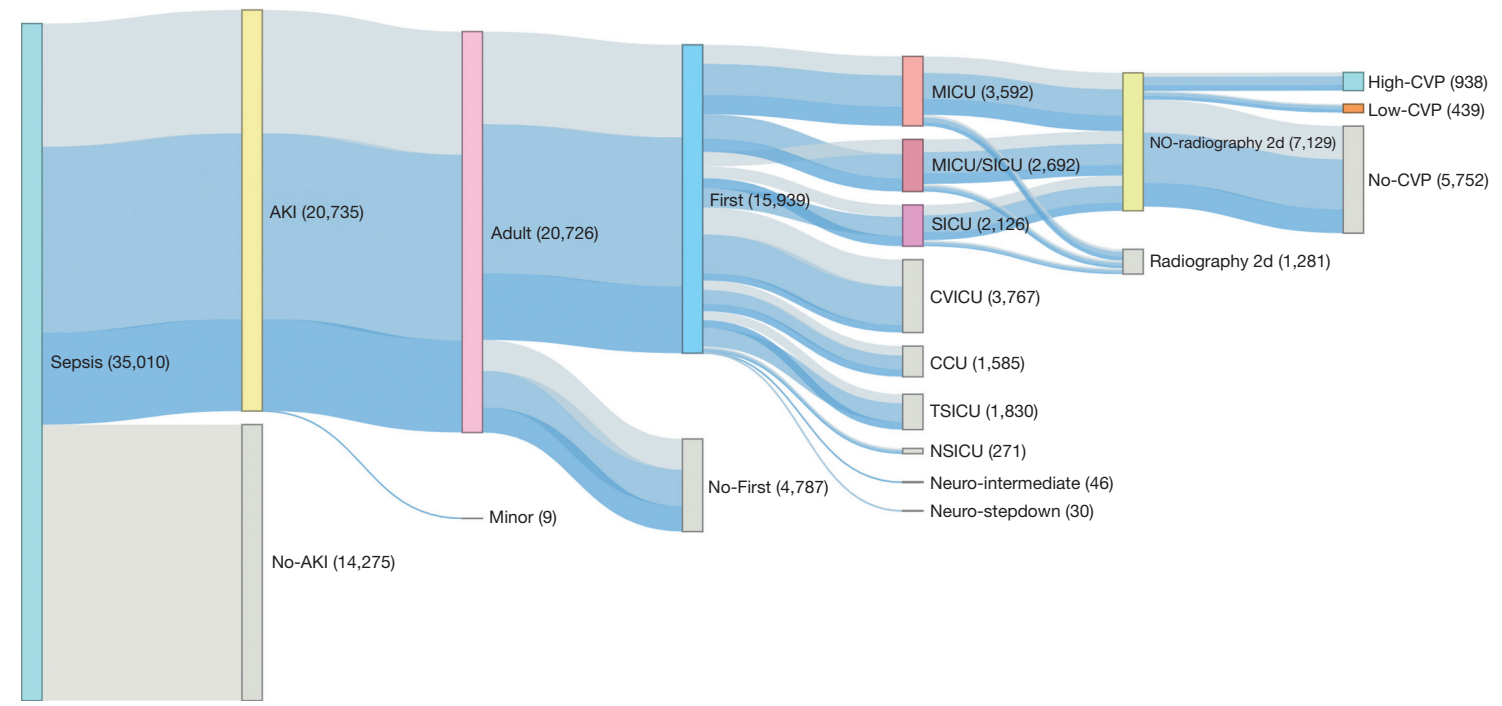

Figure 1 Inclusion/exclusion criteria used to select the final cohort of 1,377 patients. AKI, acute kidney injury; MICU, Medical Intensive Care Unit; SICU, Surgical Intensive Care Unit; CVICU, Cardiac Vascular Intensive Care Unit; CCU, Coronary Care Unit; TSICU, Trauma Surgical Intensive Care Unit; NSICU, Neurosurgery Intensive Care Unit; CVP, central venous pressure.

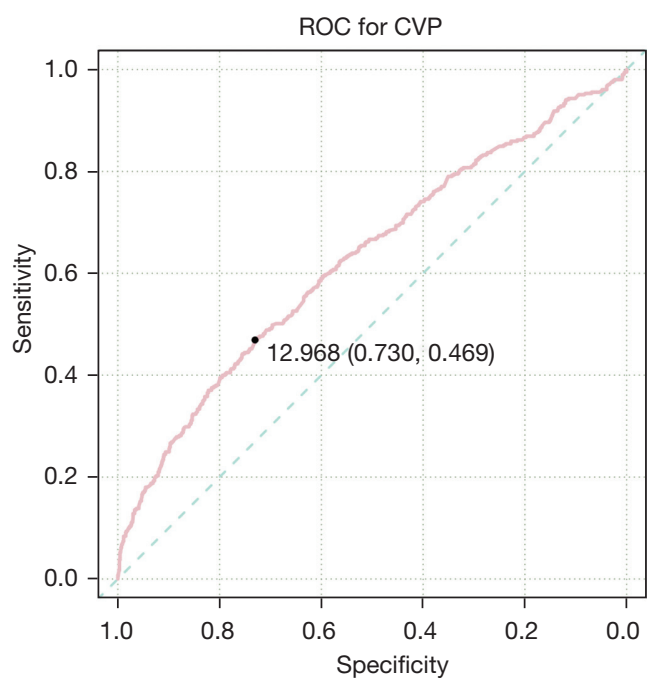

Figure 2 ROC curves for clinical outcome and CVP measurements. ROC, receiver operating characteristic; CVP, central venous pressure.

these assays were obtained as covariates.

\section{Results}

\section{Grouping of patients and general information}

A total of 35,010 records from the MIMIC-IV database that met the definition of sepsis were extracted. The following records were excluded: (I) 14,275 records that did not meet the KDIGO criteria; (II) nine records aged $\leq 18$ years; (III) 4,787 records that did not meet the criteria for the first time; (IV) 7,529 patients were excluded for their care unit were not included in (Medical) MICU or SICU or both of them; (V) 1,281 records of contrast performed within $48 \mathrm{~h}$ before meeting the KDIGO criteria; and (VI) 5,752 records with no CVP data. Finally, 1,377 patients were included in this study (Figure 1).

Through ROC analysis of the mean CVP after enrollment (Figure 2), we determined that the best cutoff value was $12.968 \mathrm{mmHg}(0.730,0.469)$. We selected $13 \mathrm{mmHg}(0.731,0.464)$ as the cut-off value for patient grouping and divided all patients into a high CVP group $(n=449)$ and a low CVP group $(n=928)$. The clinical information of both groups is shown in Table 1.

\section{Propensity scores}

We used the GBM to construct the propensity score model employing the 39 covariates (19). Matching was performed 1:1 to finally obtain a matched cohort of 288 patients (Table 1, Figure 3). In the matched cohort, the low CVP group had a lower 28 -day mortality (19.4\% vs. 34.7\%) (OR: 0.454; 95\% CI: 0.263, 0.771), and lower initial CVP levels. In addition, the high CVP group had more patients with a 
Table 1 Comparison of the clinical information between the original and matched cohorts

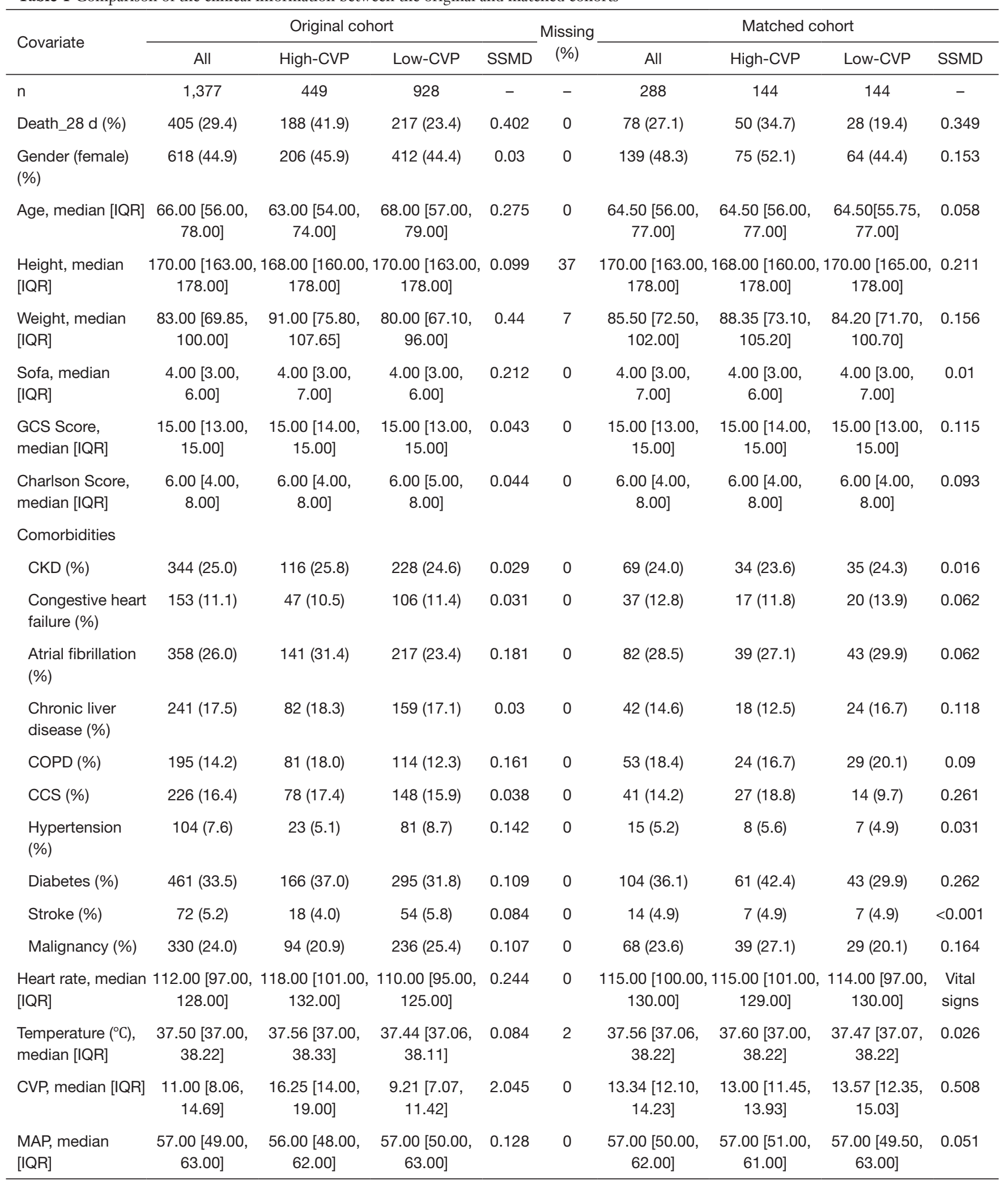

Table 1 (continued) 
Table 1 (continued)

\begin{tabular}{|c|c|c|c|c|c|c|c|c|c|}
\hline Covariate & \multicolumn{4}{|c|}{ Original cohort } & $\begin{array}{c}\text { Missing } \\
(\%)\end{array}$ & \multicolumn{4}{|c|}{ Matched cohort } \\
\hline \multicolumn{10}{|l|}{ Laboratory tests } \\
\hline $\begin{array}{l}\text { WBC, median } \\
{[\text { IQR] }}\end{array}$ & $\begin{array}{c}13.70[9.20 \\
19.90]\end{array}$ & $\begin{array}{c}14.25[9.40 \\
21.45]\end{array}$ & $\begin{array}{c}13.50[9.05 \\
19.30]\end{array}$ & 0.052 & 3 & $\begin{array}{c}14.00[10.10, \\
21.70]\end{array}$ & $\begin{array}{c}14.90[10.40 \\
22.30]\end{array}$ & $\begin{array}{c}12.95[9.40 \\
21.15]\end{array}$ & 0.002 \\
\hline $\begin{array}{l}\text { Platelet, median } \\
{[\mathrm{IQR}]}\end{array}$ & $\begin{array}{c}149.00[82.00 \\
232.75]\end{array}$ & $\begin{array}{c}153.00[83.00, \\
224.50]\end{array}$ & $\begin{array}{c}146.00[82.00, \\
235.00]\end{array}$ & 0.033 & 3 & $\begin{array}{c}154.00[87.50, \\
239.50]\end{array}$ & $\begin{array}{c}165.00[97.00 \\
240.00]\end{array}$ & $\begin{array}{c}135.00[81.50 \\
235.75]\end{array}$ & 0.213 \\
\hline $\begin{array}{l}\text { ALB, median } \\
{[\text { IQR] }}\end{array}$ & $\begin{array}{l}2.70[2.30 \\
\quad 3.00]\end{array}$ & $\begin{array}{l}2.70[2.30 \\
\quad 3.10]\end{array}$ & $\begin{array}{l}2.60[2.30 \\
\quad 3.00]\end{array}$ & 0.068 & 53 & $\begin{array}{l}2.60[2.30, \\
\quad 3.00]\end{array}$ & $\begin{array}{l}2.50[2.20 \\
\quad 3.00]\end{array}$ & $\begin{array}{l}2.60[2.30 \\
\quad 3.10]\end{array}$ & 0.171 \\
\hline $\begin{array}{l}\text { Bun, median } \\
{[\mathrm{IQR}]}\end{array}$ & $\begin{array}{c}34.00[21.50, \\
52.00]\end{array}$ & $\begin{array}{c}38.00[23.00 \\
58.00]\end{array}$ & $\begin{array}{c}32.00[21.00, \\
49.00]\end{array}$ & 0.212 & 1 & $\begin{array}{c}35.00[23.00, \\
53.00]\end{array}$ & $\begin{array}{c}34.00[22.50 \\
52.00]\end{array}$ & $\begin{array}{c}36.50[24.25 \\
56.00]\end{array}$ & 0.105 \\
\hline $\begin{array}{l}\text { Lactate, median } \\
{[\mathrm{IQR}]}\end{array}$ & $\begin{array}{l}2.50[1.50 \\
\quad 4.70]\end{array}$ & $\begin{array}{c}3.15[1.70 \\
5.73]\end{array}$ & $\begin{array}{c}2.30[1.42, \\
\quad 4.38]\end{array}$ & 0.365 & 27 & $\begin{array}{c}3.10[1.60, \\
\quad 5.20]\end{array}$ & $\begin{array}{c}3.40[1.50 \\
5.30]\end{array}$ & $\begin{array}{c}3.00[1.72, \\
\quad 5.00]\end{array}$ & 0.194 \\
\hline $\begin{array}{l}\text { P/F, median } \\
{[\mathrm{IQR}]}\end{array}$ & $\begin{array}{c}116.00[77.00, \\
182.00]\end{array}$ & $\begin{array}{c}90.53[67.00 \\
148.00]\end{array}$ & $\begin{array}{c}131.83[86.00, \\
194.75]\end{array}$ & 0.456 & 25 & $\begin{array}{c}102.93[73.00, \\
164.00]\end{array}$ & $\begin{array}{c}94.29[71.00 \\
150.98]\end{array}$ & $\begin{array}{c}108.00[74.00, \\
170.00]\end{array}$ & 0.125 \\
\hline $\begin{array}{l}\mathrm{PCO}_{2}, \text { median } \\
{[\mathrm{IQR}]}\end{array}$ & $\begin{array}{c}44.00 \\
{[37.00,51.00]}\end{array}$ & $\begin{array}{c}47.00 \\
{[39.00,55.00]}\end{array}$ & $\begin{array}{c}43.00 \\
{[37.00,49.00]}\end{array}$ & 0.311 & 2 & $\begin{array}{c}47.00 \\
{[39.00,54.00]}\end{array}$ & $\begin{array}{c}48.00 \\
{[40.00,55.00]}\end{array}$ & $\begin{array}{c}45.00 \\
{[37.00,53.00]}\end{array}$ & 0.119 \\
\hline \multicolumn{10}{|l|}{ Interventions } \\
\hline CRRT (\%) & $81(5.9)$ & $39(8.7)$ & $42(4.5)$ & 0.168 & 0 & $20(7.0)$ & $7(4.9)$ & $13(9.1)$ & 0.165 \\
\hline $\begin{array}{l}\text { Mechanical } \\
\text { ventilation (\%) }\end{array}$ & $695(50.5)$ & $232(51.7)$ & 463 (49.9) & 0.036 & 0 & $156(54.2)$ & $71(49.3)$ & $85(59.0)$ & 0.196 \\
\hline Vasopressor (\%) & $533(38.7)$ & $202(45.0)$ & $331(35.7)$ & 0.191 & 0 & $124(43.1)$ & $64(44.4)$ & $60(41.7)$ & 0.056 \\
\hline Sedation (\%) & $568(41.2)$ & $183(40.8)$ & 385 (41.5) & 0.015 & 0 & $118(41.0)$ & $59(41.0)$ & $59(41.0)$ & $<0.001$ \\
\hline Analgesia (\%) & $589(42.8)$ & $191(42.5)$ & 398 (42.9) & 0.007 & 0 & $114(39.6)$ & 57 (39.6) & 57 (39.6) & $<0.001$ \\
\hline
\end{tabular}

CS, Glasgow Coma Scale; CKD, chronic kidney disease; COPD, chronic obstructive pulmonary disease; CCS, chronic ischemic heart disease; CVP, central venous pressure; SSMD, standardized mean difference; MAP, mean arterial pressure; WBC, white blood cell; ALB, albumin; Tbil, total bilirubin; Cre, creatinine; Bun, blood urea nitrogen; BNP, brain natriuretic peptide; CRRT, continuous renal replacement therapy. 


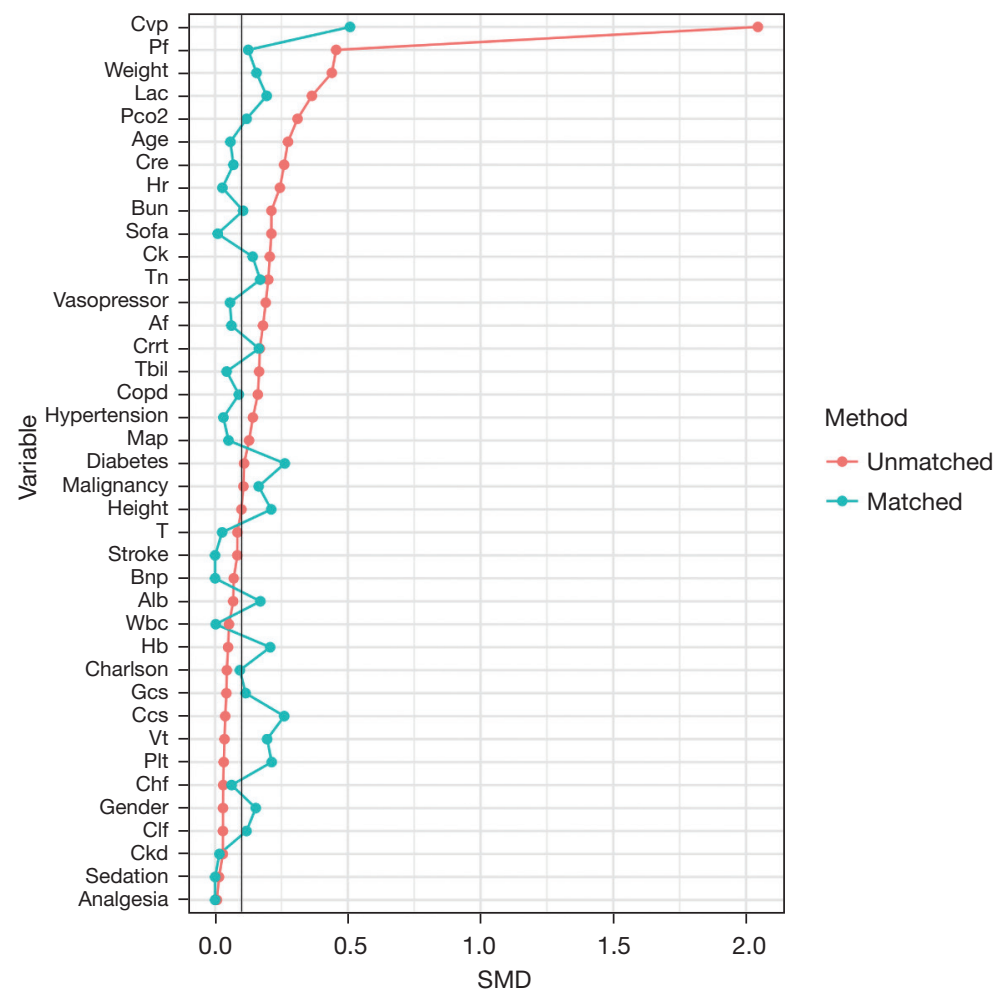

Figure 3 The relationship between SMD and 39 covariates before and after the propensity score matching model. Cvp, central venous pressure; Pf, oxygenation index; Cre, creatinine; Hr, heart rate; Bun, blood urea nitrogen; Sofa, Sequential Organ Failure Assessment; $\mathrm{Ck}$, creatinine kinase; Tn, troponin; Af, atrial fibrillation; Crrt, continuous renal replacement therapy; Tbil, total bilirubin; Copd, chronic obstructive pulmonary disease; Map, mean arterial pressure; T, temperature; Bnp, brain natriuretic peptide; Alb, Albumin; Wbc, white blood cell; Hb, hemoglobin; Gcs, Glasgow Coma Scale Score; Ccs, chronic ischemic heart disease; Vt, mechanical ventilation; Plt, platelet; Chf, congestive heart failure; Clf, chronic liver disease; Ckd, chronic kidney disease; SMD, standardized mean difference.

history of chronic coronary syndrome (CCS) $[27(18.8 \%)$ vs. $14(9.7 \%)$ ] and diabetes mellitus [61 (42.4\%) vs. 43 (29.9\%)].

\section{Sensitivity analysis}

In the matched cohort, most of the weighted variables had been balanced between the two groups. Only three variables remained: history of CCS before admission, history of diabetes mellitus, and CVP level. A regression model was developed to adjust for these unbalanced covariates on the weighted cohort in a doubly robust estimation framework. After correction for confounders, low CVP was identified as an independent protective factor for patient prognosis ( $\mathrm{P}<0.05$, OR: 0.434; 95\% CI: 0.244, 0.757).

Using the propensity score method, the propensity score IPW was used to standardize the differences between these two cohorts (20). Low CVP was also a protective factor for patient prognosis in the IPW model $(\mathrm{P}<0.05$, OR: 0.547;
95\% CI: 0.454, 0.658).

In addition, low CVP was also found to be an independent protective factor for patient prognosis in a multifactorial regression of the original cohort $(\mathrm{P}<0.05$, OR: 0.352; 95\% CI: 0.127, 0.932).

The above method of analysis revealed a very significant result, with the two groups showing a significant difference in mortality. The propensity score-matched mortality rates for the upper and lower CVP groups were $41.9 \%$ vs. 23.4\%. As shown in Figure 4, the same conclusion was reached by four statistical methods. Lower CVP is a protective factor affecting the prognosis of this group of patients.

\section{Discussion}

The advent of electronic databases has made it possible to conduct more retrospective studies (21). In an era when patient information was not available electronically, the 


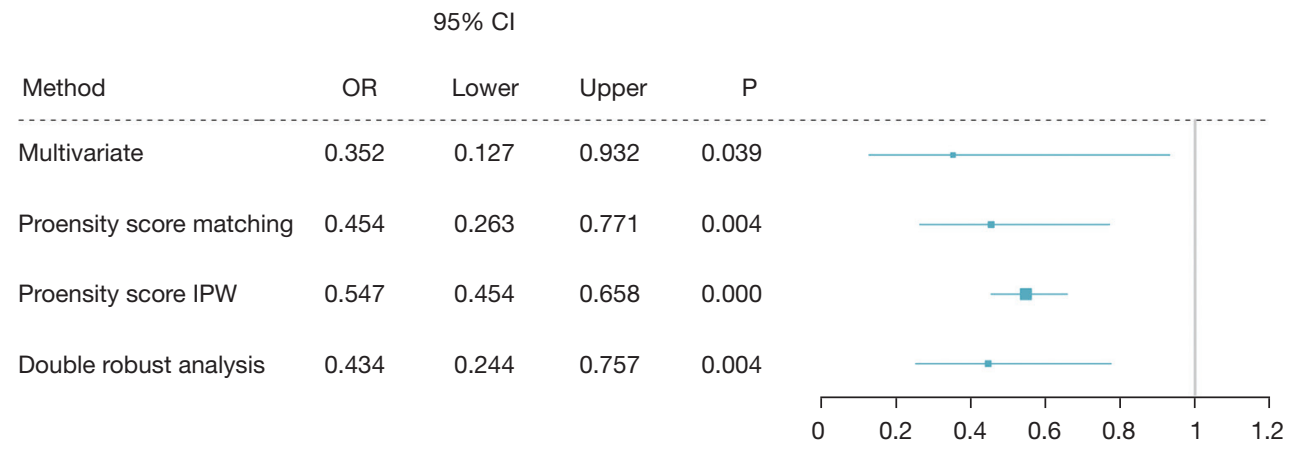

Figure 4 Primary outcome analysis with different models: multivariate logistic regression model propensity score matching model, propensity score IPW model, and double robust analysis. CI, confidence interval; OR, odds ratio; IPW, inverse probability weighting.

absence of laboratory results, vital signs, and untimely observation of patient outcomes were barriers to retrospective analyses. By using electronic databases, patient information is retained and patient privacy is well protected through appropriate means. The sheer volume and richness of the data has helped considerably in the development of more comprehensive studies.

At present, various high-end medical devices are playing an increasingly important role in the ICU wards, to the benefit of many patients. A variety of organ support and replacement strategies have provided valuable time for the recovery of organ function in critically ill patients, and have also made possible the recovery of organ function in numerous diseases with serious complications, such as sepsis and other affected organs (22).

CVP monitoring has long been an important indicator in ICU wards (23), perhaps because people are accustomed to accepting the long-standing perception of CVP, and therefore, directly taken the view that higher levels of CVP affect organ perfusion pressure when faced with critical conditions such as sepsis as granted. According to current Sepsis and KDIGO guidelines, SA-AKI has corresponding diagnostic and grading criteria, and the impact of CVP levels on SA-AKI, as a life-threatening and important complication, should be clarified.

In this study, we analyzed data on basic information, past medical history, vital signs, laboratory tests, and interventions in patients with SA-AKI, and concluded that lower levels of CVP were independently associated with decreased mortality in these patients. In addition, patients in the high CVP group tends to have previous CCS $(18.8 \%$ vs. $9.7 \%$ ) and diabetic ( $42.4 \%$ vs. $29.9 \%)$, considering that patients with chronic slow coronary syndrome and diabetic patients have poorer vascular conditions and are less able to regulate the hemodynamic changes produced by sepsis (24). As a result, it is also consistent with the finding that the high CVP group had higher mortality. The vital signs in this study were selected as the mean of the 24-hour values before and after the diagnosis of sepsis. Notably, the mean CVP level in the high CVP group was slightly lower than that in the low CVP group, which may be related to the low CVP level at the time of ICU admission in this group of patients, as the CVP was not yet at a very high level at the time of ICU admission. Therefore, the prime CVP was ignored during the subsequent treatment,thus follow-up changes in CVP can lead to imparied organ function damage (25), leading to a higher mortality rate in patients with low levels of CVP at the time of transfer instead. In contrast, patients with higher CVP levels at the time of transfer may have a better prognosis due to the proper control of hemodynamic indices and the avoidance of further organ damage (26). The limitations of this study are that there were more missing CVP values in the 1,377 included patients, which may have had some impact on the results. Also, this study used the Sepsis 3 diagnostic criteria, and the diagnosis of sepsis is currently still clinically dependent, which may also have had an impact on the results. Thus, further studies are still needed to explore this.

Although the pathogenesis of sepsis remains to be explored, mitigating the organ damage associated with its complications is also an important part of sepsis treatment (27). In the absence of effective treatments, we have to rely on early detection of early sepsis patients who have not yet progressed to a severe state as well as early control of factors that may be associated with exacerbation of the disease, thereby saving organ function. Although control of CVP levels is a proven therapeutic measure (28), it may often be overlooked in clinical work because of 
the poor circulatory status of septic patients (29), and the depletion of the organism by a severe infectious state makes the systemic hemodynamic status more difficult to control. However, in clinical work, attention should still be paid to $\mathrm{CVP}$, in addition to prioritizing the protection of organ function and the reduction of microcirculatory afterload.

This study has some limitations. First, it is a retrospective study and several biases, including selection bias, should be considered. Second, data were recorded in a long study period; clinical practice is evolving quickly and older management strategies may be different from current practices. Third, lower breakpoints of CVP are usually considered in standard clinical practice and findings cannot be generalized for CVP of $10 \mathrm{cmH}_{2} \mathrm{O}$ or lower. Lastly, due to the limitation of the database, there is no contents about some important variables such as echography associated with these patients; thus lower cava diameter was not be measured in our study and the timing of RRT was not explored in the research either due to the same reason. Therefore, CVP alone cannot be extrapolated to make decisions at the bedside. In patients with high CVP, it is needed to rule out right heart dysfunction. That is common in diabetes \& cardiomyopathy. Data were not adjusted for these two confounding variables. High CVP with cava dilation is cardio-renal type III AKI and requires urgent RRT. Need to differentiate from high CVP with cava diameter normal, which requires vasoactive agents in clinical practice.

\section{Conclusions}

In summary, lower CVP levels are an independent protective factor affecting patient prognosis in patients with SA-AKI. In patients with high CVP, it is needed to rule out cardiac dysfunction and lower cava diameter needs to be measured with echography to guide clinical management.

\section{Acknowledgments}

The authors appreciate the academic support from the AME Radiology Collaborative Group.

Funding: None.

\section{Footnote}

Reporting Checklist: The authors have completed the STROBE reporting checklist. Available at https://atm. amegroups.com/article/view/10.21037/atm-22-588/rc
Conflicts of Interest: All authors have completed the ICMJE uniform disclosure form (available at https://atm. amegroups.com/article/view/10.21037/atm-22-588/coif). The authors have no conflicts of interest to declare.

Ethical Statement: The authors are accountable for all aspects of the work in ensuring that questions related to the accuracy or integrity of any part of the work are appropriately investigated and resolved. The study was conducted in accordance with the Declaration of Helsinki (as revised in 2013).

Open Access Statement: This is an Open Access article distributed in accordance with the Creative Commons Attribution-NonCommercial-NoDerivs 4.0 International License (CC BY-NC-ND 4.0), which permits the noncommercial replication and distribution of the article with the strict proviso that no changes or edits are made and the original work is properly cited (including links to both the formal publication through the relevant DOI and the license). See: https://creativecommons.org/licenses/by-nc-nd/4.0/.

\section{References}

1. Singer M, Deutschman CS, Seymour CW, et al. The Third International Consensus Definitions for Sepsis and Septic Shock (Sepsis-3). JAMA 2016;315:801-10.

2. Fleischmann C, Scherag A, Adhikari NK, et al. Assessment of Global Incidence and Mortality of Hospital-treated Sepsis. Current Estimates and Limitations. Am J Respir Crit Care Med 2016;193:259-72.

3. Prest J, Sathananthan M, Jeganathan N. Current Trends in Sepsis-Related Mortality in the United States. Crit Care Med 2021;49:1276-84.

4. Luhr R, Cao Y, Söderquist B, et al. Trends in sepsis mortality over time in randomised sepsis trials: a systematic literature review and meta-analysis of mortality in the control arm, 2002-2016. Crit Care 2019;23:241.

5. Peerapornratana S, Manrique-Caballero CL, Gómez H, et al. Acute kidney injury from sepsis: current concepts, epidemiology, pathophysiology, prevention and treatment. Kidney Int 2019;96:1083-99.

6. Gullo A, Bianco N, Berlot G. Management of severe sepsis and septic shock: challenges and recommendations. Crit Care Clin 2006;22:489-501, ix.

7. Angus DC, van der Poll T. Severe sepsis and septic shock. N Engl J Med 2013;369:840-51.

8. Chawla LS, Eggers PW, Star RA, et al. Acute kidney injury 
and chronic kidney disease as interconnected syndromes. N Engl J Med 2014;371:58-66.

9. Poston JT, Koyner JL. Sepsis associated acute kidney injury. BMJ 2019;364:k4891.

10. Bellomo R, Kellum JA, Ronco C, et al. Acute kidney injury in sepsis. Intensive Care Med 2017;43:816-28.

11. Godin M, Murray P, Mehta RL. Clinical approach to the patient with AKI and sepsis. Semin Nephrol 2015;35:12-22.

12. Bagshaw SM, Uchino S, Bellomo R, et al. Septic acute kidney injury in critically ill patients: clinical characteristics and outcomes. Clin J Am Soc Nephrol 2007;2:431-9.

13. Manrique-Caballero CL, Del Rio-Pertuz G, Gomez H. Sepsis-Associated Acute Kidney Injury. Crit Care Clin 2021;37:279-301.

14. Kidney International Supplements. Kidney Int Suppl 2012;2:1. Available online: https://kdigo.org/guidelines/ acute-kidney-injury/

15. Sood MM, Shafer LA, Ho J, et al. Early reversible acute kidney injury is associated with improved survival in septic shock. J Crit Care 2014;29:711-7.

16. Chen X, Wang X, Honore PM, et al. Renal failure in critically ill patients, beware of applying (central venous) pressure on the kidney. Ann Intensive Care 2018;8:91.

17. Johnson A, Bulgarelli L, Pollard T, et al. MIMICIV (version0.4). PhysioNet 2020. Available online: https://doi. org/10.13026/a3wn-hq05

18. Kallner A. Bayes' theorem, the ROC diagram and reference values: Definition and use in clinical diagnosis. Biochem Med (Zagreb) 2018;28:010101.

19. Fortin SP, Johnston SS, Schuemie MJ. Applied comparison of large-scale propensity score matching and cardinality matching for causal inference in observational research.

Cite this article as: Huo Y, Wang X, Li B, Rello J, Kim WY, Wang $\mathrm{X}, \mathrm{Hu} Z$. Impact of central venous pressure on the mortality of patients with sepsis-related acute kidney injury: a propensity score-matched analysis based on the MIMIC IV database. Ann Transl Med 2022;10(4):199. doi: 10.21037/atm22-588
BMC Med Res Methodol 2021;21:109.

20. Cole SR, Hernán MA. Constructing inverse probability weights for marginal structural models. Am J Epidemiol 2008;168:656-64.

21. Beam AL, Kohane IS. Big Data and Machine Learning in Health Care. JAMA 2018;319:1317-8.

22. Zarbock A, Kellum JA, Schmidt C, et al. Effect of Early vs Delayed Initiation of Renal Replacement Therapy on Mortality in Critically Ill Patients With Acute Kidney Injury: The ELAIN Randomized Clinical Trial. JAMA 2016;315:2190-9.

23. Kipnis E, Ramsingh D, Bhargava M, et al. Monitoring in the intensive care. Crit Care Res Pract 2012;2012:473507.

24. Advani A. Acute Kidney Injury: A Bona Fide Complication of Diabetes. Diabetes 2020;69:2229-37.

25. Rajendram R, Prowle JR. Venous congestion: are we adding insult to kidney injury in sepsis? Crit Care 2014;18:104.

26. Chen KP, Cavender S, Lee J, et al. Peripheral Edema, Central Venous Pressure, and Risk of AKI in Critical Illness. Clin J Am Soc Nephrol 2016;11:602-8.

27. Vellinga NA, Ince C, Boerma EC. Elevated central venous pressure is associated with impairment of microcirculatory blood flow in sepsis: a hypothesis generating post hoc analysis. BMC Anesthesiol 2013;13:17.

28. Badin J, Boulain T, Ehrmann S, et al. Relation between mean arterial pressure and renal function in the early phase of shock: a prospective, explorative cohort study. Crit Care 2011;15:R135.

29. De Backer D, Donadello K, Taccone FS, et al. Microcirculatory alterations: potential mechanisms and implications for therapy. Ann Intensive Care 2011;1:27. 